\title{
A Graph Based Routing Algorithm for Multi-hop Cognitive Radio Networks
}

\author{
Yogesh R Kondareddy \\ Auburn University \\ Auburn, AL 36830 \\ kondayr@auburn.edu
}

\author{
Prathima Agrawal \\ Auburn University \\ Auburn, AL 36830 \\ agrawpr@auburn.edu
}

\begin{abstract}
Cognitive radio networks that allow dynamic spectrum access are considered spectrally more efficient than networks using fixed spectral allocation. These networks are characterized by dynamically changing channel sets at each node. The graph theoretic approach used in traditional multi-hop networks fails to efficiently model multi-hop cognitive radio networks and capture the required information for optimal routing. Hence, conventional graph-based routing protocols such as DSR or AODV cannot be used directly, for route discovery in such networks. In this paper, a unique multi-edge planar graph model for routing in such type of networks is proposed, which is quite simple and could be used in conjunction with any conventional graph-based routing protocol. The model is validated through simulations and the complexity of the model is shown to be lesser than an earlier layered graph model.
\end{abstract}

\section{Categories and Subject Descriptors}

C.2.1 [Computer-Communication Networks]: Network Architecture and Design - Network topology.

C.2.2 Network Protocols - Routing protocols.

\section{General Terms}

Algorithms, Design, Standardization, Verification.

\section{Keywords}

Cognitive radio networks, multiple edge graph, routing.

\section{INTRODUCTION}

A cognitive network is an opportunistic network. Spectrum opportunity deals with the usage of an available (free) channel that is a part of the spectrum which is not currently used by primary users [1]. The licensed owner of a frequency band is called a primary user and the one who utilizes spectrum opportunities for communication is called a secondary user. When the receiver is not in the transmitting range of the sender, data is forwarded through several hops forming a Multi-Hop Cognitive Radio Network (MHCRN). But unlike in a normal multi-hop network in which all users operate in the same channel, users in a MHCRN use different frequencies depending on spectrum availability. As a result, two users are connected depending on whether they have a common frequency band for operation.

While the MHCRN concept appears attractive, there are many complexities introduced not only due to the cognitive PHY layer but also due to multi-hop characteristics of these networks. Some of them are: since the available channels for communication vary with primary user's traffic, the cognitive PHY layer is dynamic in nature. Due to the multi-hop nature, the choice of a channel for each secondary user is now constrained by the available channel set at every user along the route. A route is possible only if every pair of users along the route have at least one common channel available at their respective locations. After a communication path is established through a set of intermediate nodes, still the route may fail due to the dynamic nature of the PHY layer at each node. A new route has to be discovered with the new set of available channels. Due to the abrupt failure in communications and delays caused in discovering a new route, traditional routing protocols in the network stack are either not applicable or very inefficient on MHCRNs. This is due to the fact that the graph theoretic approach which is very efficient in modeling traditional networks, fails to efficiently model the cognitive network environment. This paper proposes the idea of multi-edge planar graphs to model MHCRNs. Routing operations in MHCRN are conveniently analyzed using this model in combination with traditional routing protocols such as AODV [2], DSDV [3], and DSR [4].

The rest of the paper is organized as follows: routing issues are discussed in section II. The new multi-edge planar graph model is presented in Section III. The graph model's performance in routing in MHCRN is evaluated in Section IV using a custom simulator written in $\mathrm{C}$ with conclusion in Section $\mathrm{V}$.

\section{ROUTING ISSUES AND RELATED WORK}

Routing in multi-hop networks has been extensively studied in the literature. Several protocols have been proposed among which AODV [2], DSDV [3], DSR [4] are some of the widely used ones. Most of these protocols are graph based protocols, wherein a network is represented as a graph. Nodes in the graph represent the users and edges are used to join two nodes which can communicate with each other. The edges are assigned weights which generally represent the distance from one node to another. Protocols, mentioned above use this graph based information and find routes between nodes using various metrics, like DSDV finds a route based on the shortest distance or hop count. Hence, the graph theoretic approach is very efficient in modeling traditional networks and provides sufficient information for route discovery.

Traditional wireless networks make two general but fundamental assumptions.

- $\quad$ Fixed spectrum allocation i.e., pair of nodes communicate over a fixed band of spectrum (channel) and

- That the fixed channel is always available for communication.

This means that the physical layer and its fundamental characteristics like bandwidth, and channel of operation do not change and are static both in space and time. As a result of these general assumptions, the graph model mentioned above is sufficient to represent traditional networks.

\footnotetext{
* Conference name: WICON'08, November 17-19, 2008, Maui, Hawaii, USA.

* Copyright number: Copyright 2008 ICST 978-963-9799-36-3.
} 
In a MHCRN, the availability of a particular channel depends on primary user's traffic. So the PHY layer of a MHCRN is dynamic and may vary in characteristics like bandwidth, power constraints and interference levels. If a route discovery has to be initiated in such networks, a series of steps have to be followed in which, scanning for available channels at each node, deciding on a common channel for each pair of neighbors are most important ones. If there is more than one common channel between a pair of nodes, then an optimal channel has to be chosen based on various criterion such as interference level, bandwidth etc. Moreover, due to different transmission power levels for different frequency bands (imposed by FCC), channels may support varied transmission ranges [5] due to which a node reachable in one channel may not be reachable in another. Hence, the neighbor set of a node may depend on the type of channel used for communication. Due to lack of consideration of important information like available channel set at each node, possible routes to other nodes and neighbor set in each channel, traditional graph model is insufficient to model MHCRNs.

A layered graph model was proposed as a solution to this problem in [6]. In this graph model, each channel or frequency of operation is represented by a layer of the graph. Each layer is a sub-graph containing nodes representing users. The nodes in each sub-graph are connected if they are in the transmission range of each other in that particular channel. Communication among the nodes across layers is possible through inter-layer links. Using this graph model, a routing strategy was built to find a near optimal route [6].

The disadvantages of this graph model are that the model is complex to represent. Traditional routing protocols like AODV [2], DSDV [3] and DSR [4] assume planar structured graphs; as a result a direct application of these protocols on layered graph model is not possible.

Hence a 'Multi-Edge Planar Graph Model for Routing in MHCRNs' is proposed in this paper which embeds the dynamic behavior of a MHCRN into its edge weights. Such a model is simpler and also accurate as it takes into account various radio channel related parameters in determining edge weights. The next two sections discuss the model and evaluate its performance.

\section{MULTI-EDGE GRAPH MODEL}

As the name implies, this graph model, unlike the traditional graph models used in routing, has multiple edges between a pair of nodes. This added feature of the graph accounts for the modeling of extra characteristics of MHCRNs such as available channel set, possible routes to other nodes through various channels and neighbor set of each channel. Unlike in layered graph model where layers represent different channels, multi-edged graph model takes advantage of extra edges to represent the channels and weights of these edges to represent the channel's characteristics. Due to this characteristic of multi-edged graph model, a layered structure is obviated and is reduced to a planar structure. It can be argued that the layer complexity is shifted to edge complexity in the multiedge planar graph model. But it will be demonstrated in section IV that edge complexity of the new model is much lower than layered graph model. This section explains the proposed model in detail.

\section{A. Graph Topology Formation}

In MHCRN, routing depends on the available channels which in turn depend on the primary user's traffic. This means that for an optimal choice of the route, the routing layer should be provided with information about the available channel set at each node by the PHY layer. Additionally, the total number of interfaces available at each node is also important for optimal choice of the route. For example, if an intermediate node in the route has more than one interface, it can choose two different channels for incoming and outgoing communication, so that the interference is reduced and throughput is increased [7]. So a cross layer design is necessary to keep the routing layer updated about the changes in the PHY layer. It is assumed in the rest of the paper that the information about the total number of interfaces and available channels at each node is provided to the routing layer of the nodes in question.

In the Multi-Edge planar graph model, each user is represented as a Node in the graph. Each channel is represented by an Edge. Let graph $G$ denote the multi-edged graph, $N$ denote the set of nodes and $C$ denote the set of all possible channels. So a pair of nodes can have 2 edges if they can use two different frequencies (channels) for communication unlike conventional graph models where each pair of nodes has only 1 channel in common as in [7]. For example if nodes A and B have two channels to communicate, then it is represented as shown in Fig. 1. A and B can communicate through channel 1 and channel 2. Therefore, nodes A and B are connected by two edges. W1 and W2 represent the weights of the edges in each channel which can be simple distance or a complex combination of different factors like interference, energy etc. Every channel is assigned a channel ID. For example Channel 1 is assigned ' 1 ' as channel ID.

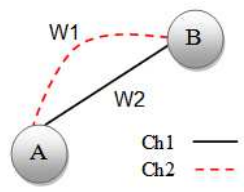

Figure 1. Nodes A and B linked by 2 edges.

Now, consider a graph with 4 nodes and 3 different channels. Each node may have different channels available with it. Two nodes in this graph are connected if they have a common channel in their channel sets. Graphical representation of these 4 nodes in a traditional network with fixed channel allocation is shown in Fig. $2 \mathrm{a}$. Since all the nodes can communicate in only one channel in such networks, there is only one edge between every pair of nodes which can communicate with each other. The numbers on the edges represent the weights of the edges which in this case are distances. A cognitive network where each node has varying number of channels available with it is shown in Fig. 2b. In this graph, the node pairs A-D, D-C, and B-C have channels 1 and 2 in common but the node pair A-B has channels 1, 2 and 3 in common consequently having three edges between them. All edges between every pair of nodes are assigned the same weight because, the weights represent distance between pair of nodes and it does not vary with type of channel. So, any routing protocol such as AODV [2], DSDV [3], and DSR [4] gives the shortest route as an optimal route.

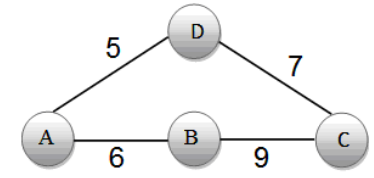

a)

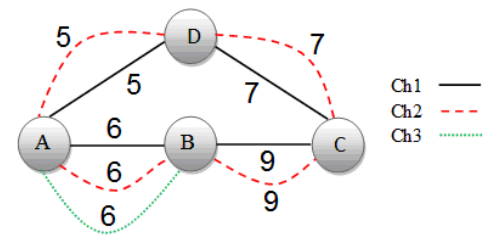

b)
Figure 2a. Graphical representation in traditional network. 2b. Graphical representation in cognitive network. 


\section{B. Weight assignment and Routing}

All graph based protocols, find a route based on the weights of the edges of the graph. If the weights of the edges represent the distance between the nodes, a graph based protocol like DSDV would choose the shortest path route. If the weights were a function of interference then the route with lowest interference is chosen. Therefore weight assignment is a very important step in building a graph model. In the multi-edged graph model a problem of interface constraint arises due to the multi-edged nature of the graph. The problem is explained below and solved by modifying the weights of the edges. Finally, the weights of all edges are added with a cognitive cost function which is explained later.

\section{Interface constraint}

A node can have a single or multiple radios. Each radio (interface) is capable of changing to a required frequency (channel) to communicate. Switching the interface between channels is feasible in a classical wireless network, though it requires fine synchronization between neighboring nodes and introduces overhead. But in a cognitive environment where the channels are not bound to $2.4 \mathrm{GHz}$ ISA band, this type of switching might not be feasible. Moreover, the channels in a cognitive network are distributed across a large spectrum and the channels may be separated with a large band. In such a scenario, it might not be practical to switch channels at packet granularity. A method to avoid the choice of routes in which switching occurs, is proposed below.

Now let us consider Fig. 2b. If the weights are a simple function of distance, then all the edges between a fixed pair of nodes would weigh the same as explained earlier. For example, weights of channels 1, 2 and 3 are the same between nodes A and B. In this case, distance vector routing might choose channel 1 between A, D and channel 2 between D, C as shown in Fig. 3. Channel used from $A$ to $D$ is different from $D$ to $C$ in this route. If node $\mathrm{D}$ were have having a single radio it should switch the interface (radio) between channels 1 and 2 for carrying on the communication. As mentioned above, it might not be always feasible, though faster radios are promised by the growing technology. Hence, there should be a way for the routing protocol to avoid choosing different channels if a node has a single available radio and when switching is not allowed. This is done by adding an Interface Constraint term to the weights of outgoing edges of single radio nodes only as shown below:

Let Interface Constraint term (IC) $=|X-Y| \infty$

$$
W=|X-Y| \infty+D
$$

Where, $\mathrm{W}$ is the Weight of the edge, $\mathrm{X}$ is the Channel ID of the incoming channel, $\mathrm{Y}$ is the Channel ID of the outgoing channel and $\mathrm{D}$ is the Distance Metric.

Since $\mathrm{X}$ and $\mathrm{Y}$ are channel ID's, if a same channel is chosen for incoming and outgoing communication, then the value of $X$ and $\mathrm{Y}$ will be equal and their difference is zero. As a result the interface constraint term is zero and the weight, $\mathrm{W}$ of the edge will just be distance, D. Suppose different channels are chosen for the incoming and outgoing communications, then mod of the difference of $\mathrm{X}$ and $\mathrm{Y}$ will be a positive non-zero value. The interface constraint term will now be infinity and the final weight, $\mathrm{W}$ would also be infinity. Hence, this option will not be considered as a successful route.

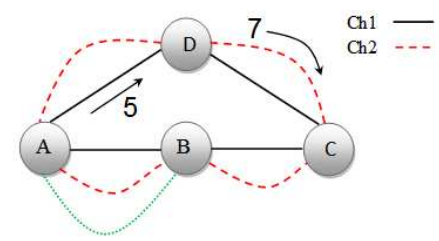

Figure 3. Path taken if only weights were considered.

Now consider the same graph as in Fig. $2 b$ and let us assume that node $\mathrm{D}$ has a single radio and it cannot communicate over two different channels. Suppose the routing protocol chose channel 1 from A to D. The weights for the next hop would be as shown below and the path followed is shown in Fig. 4.

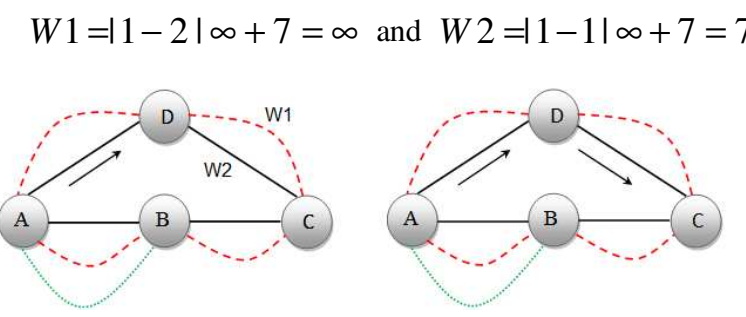

Figure 4. Path followed as the new weights are calculated

It is seen from Fig. 4 that the protocol would choose channel 1 in the second hop since the weight of the edge through channel 2 (W1) is infinity. So, by adding an interface constraint term(IC) to the weights of outgoing edges of the single radio nodes which cannot switch, the possibility of choosing two different channels by the protocol at that node is avoided.

\section{Cognitive Cost Function}

The nodes in a MHCRN have the capability to sense the channels using cognitive radio. This capability of the nodes can be used to find better routes in terms of interference. This can be done by adding an additional term to the weights of the edges, cognitive cost function (CF).

A routing cost function incorporating measurements of the instantaneous behavior of the external world, as represented for example by current network status in terms of interference suffered by overlaid networks was proposed in [8]. The cost function accounted for various terms, of which, power, multi-user interference, reliability of the channel, traffic in the channel and delay factor are important. This cost function is added to the weights of the edges to incorporate cognitive routing.

Algorithm 1 is used for the construction of the Multi-Edged graph model, weight assignment and routing in MHCRN. In the following section the performance and complexity of the multiedged graph model is studied.

Algorithm 1 Weight assignment and Routing

1) Add a node $N_{i}$ to the graph $G$ for each user in MHCRN.

2) Add an edge between between node $N_{i}$ and node $N_{j}$ if they are potential neighbors through channel $C_{i}$ for all $N_{i}, N_{j} \in N$ and $C_{i} \in C$.

3) Assign distance $\mathrm{D}$ as the weight for the edge connecting nodes $N_{i}$ and $N_{j}$ for all $N_{i}, N_{j} \in N$. 
4) Add the Interface constraint term (IC) to outgoing edges of nodes $N_{i}$ if $N_{i}$ has a single radio and can not switch for all $N_{i} \in N$.

5) Add the Cognitive cost function (CF) to the weights of all edges connecting nodes $N_{i}$ and $N_{j}$ for all $N_{i}, N_{j} \in N$.

6) Use the graph $G$ for route discovery using any graph based routing protocols like distance vector routing.

\section{SIMULATION STUDY OF MULTI- EDGED GRAPH MODEL}

A custom simulator written in $\mathrm{C}$ is used to simulate the multiedge graph. The graph model is tested using DSDV protocol. A grid topology is considered with varying number of nodes from $2 \times 2$ to $10 \times 10$. The channels available at each node are randomly selected. The probability that a particular channel is available at a node is $p$. The value of $p$ is chosen to be 0.5 . Maximum number of channels (channel set) at each node is varied from 2 to 10 . The connectivity from the first node to the last node in the graph is examined for and the percentage of successful routes is plotted. Each point on the graphs is an average of 100 simulations.

Two sets of simulations were carried out. The first set of simulations assume that all the intermediate nodes in a route can switch between channels to communicate with their two neighbors and the second set of simulations assume that no intermediate node can switch between channels in a communication. Though these assumptions are unrealistic, they serve two purposes. Firstly, they help us verify the proposed model intuitively. Secondly, they act as upper and lower bounds respectively. In practice, results will lie inbetween these results.

Fig. 5 shows the variation of percentage of successful routes with the number of nodes in the networks in which switching the interface between the channels is allowed and not allowed respectively. It is observed that there is a success rate of $90 \%$ with switching. When switching is not allowed, a node cannot communicate in two different channels simultaneously. This constraint, limits all nodes in the route to communicate using the same frequency (Channel). Because it is less probable that all nodes in the route have a common channel, the success rate is lower in such a network. When switching is possible, a route is possible if there is at least one channel between every pair of nodes along the route. As a result the percentage of successful routes is far better with switching than without switching.

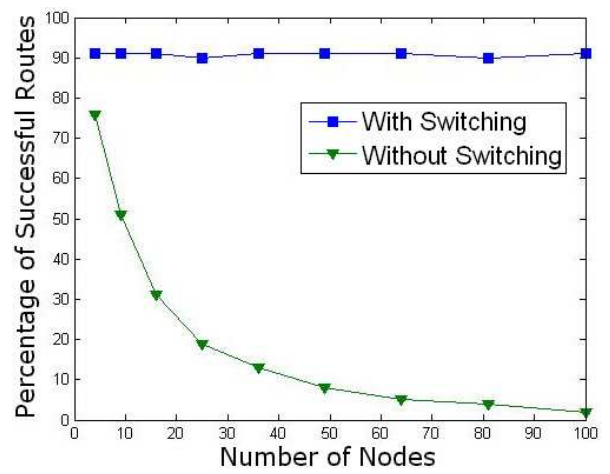

Figure 5. Comparison of percentage of successful routes as a function of number of nodes with and without switching interfaces.
It is also observed that as the number of nodes increases, the success rate decreases. This is because of the fact that with the increase in the number of nodes, the probability of finding a route with a common channel between every pair of nodes along the route decreases.

In Fig. 6, percentage of successful routes is plotted against the maximum number of channels (channel set). From this graph it is seen that with an increase in the number of channels, the success rate of the routes increases drastically to nearly $100 \%$ with switching. This is obvious from the fact that the probability of finding at least one available channel between a pair of nodes increases with the number of channels. Whereas, the probability that a common channel is found among all the nodes with increase in number of channels increases marginally.

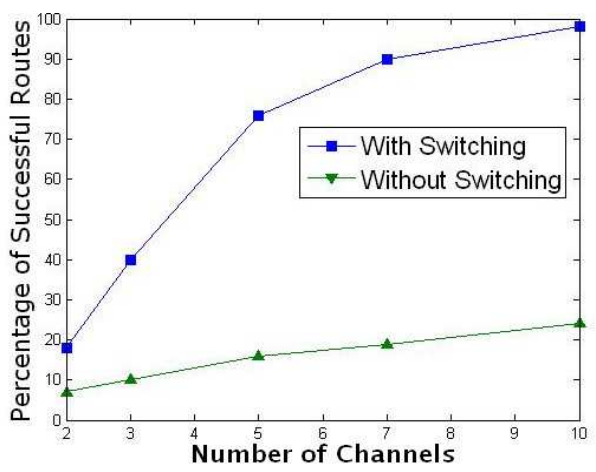

Figure 6. Comparison of percentage of successful routes as a function of number of channels with and without switching.

In a real world scenario a network consists of heterogeneous nodes. Some nodes might have single interface and some have more than one interface. When the nodes have more than one interface, they can use one interface for each incoming and outgoing channel in which case, the percentage of success rate is similar to the network in which switching is possible. So, the practical success rate lies in-between the results shown above.

\section{Analytical Model}

To verify these results an analytical model is needed to determine variation of the probability of a successful route with the number of nodes and the maximum number of channels is varied.

Probability of a successful route is derived below.

Consider a path along a chain of $n$ nodes. Probability that there exists a route between the first node and the last node if a channel is available at a particular node with a probability of $p$ is derived. Let the total number of possible channels be $c$

$n-\quad$ Number of nodes in the route

c- Number of possible channels at each node

p- Probability that a particular channel is available at a node

$P$ - Probability that a route exists between the first node and the last node

In a single radio network, each radio can communicate over only one channel at a time. So a route is possible only if all nodes in the path have at least one common channel available. 
Probability that one common channel exists among $n$ nodes

$$
=p^{n}
$$

Probability that one common channel does not exist

$$
=1-p^{n}
$$

Probability that no common channel exists among all c possible channels

$$
=\left(1-p^{n}\right)^{c}
$$

Probability that at least one common channel exists among all

possible channels

$$
\begin{aligned}
& =1-\left(1-p^{n}\right)^{c} \\
& P=1-\left(1-p^{n}\right)^{c}
\end{aligned}
$$

Thus,

In a dual/multi radio network, each node can receive and send along different channels. So, a route is possible if there is at least one common channel between the two nodes along every hop. To calculate this probability we consider each hop separately.

Probability that one common channel exists between two nodes

$$
=p^{2}
$$

Probability that a common channel does not exist between 2 nodes

$$
=1-p^{2}
$$

Probability that no common channel exists between two nodes along $\mathrm{c}$ possible channels $=\left(1-p^{2}\right)^{c}$

Probability that at least one common channel exists between two nodes $\quad=1-\left(1-p^{2}\right)^{c}$

Probability that at least one common channel exists between every hop

$$
=\left(1-\left(1-p^{2}\right)^{c}\right)^{n-1}
$$

Thus,

$$
P=\left(1-\left(1-p^{2}\right)^{c}\right)^{n-1}
$$

Since, the probability of a successful route along a path is known; the results from the graph model can be verified. The model is used to find the route possibility along the shortest path. Since grid topology was used in simulating the graph model, the shortest path would be along the diagonal. This will be a pessimistic value because, there can be other routes possible and hence the actual probability is expected to be higher. Results from the graph model and analytical model are plotted in Figs. 7 and 8.

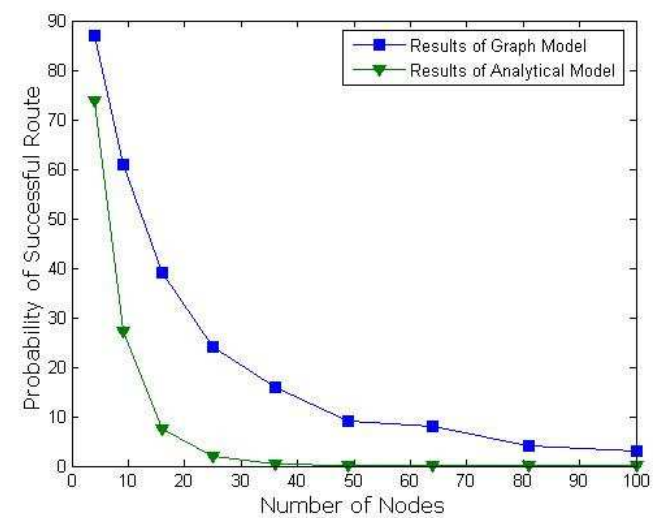

Figure 7. Comparison of Practical Results and Theoretical Results from the analytical model for a single radio network as the number of nodes are varied for a set of 10 channels

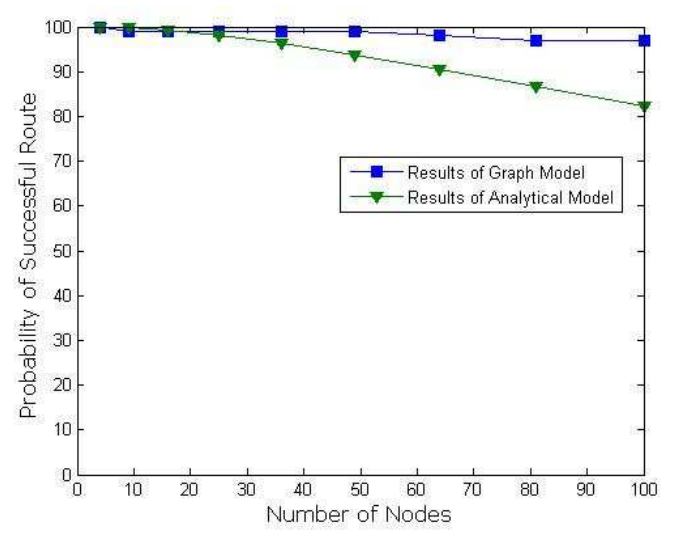

Figure 8. Comparison of Practical Results and Theoretical Results from the analytical model for a dual radio network as the number of nodes are varied for a set of 10 channels

It is seen from the simulations that the results from the graph model match the results from the analytical model. Hence, the proposed graph model is demonstrated to be valid in modeling MHCRN. Since the multi-edged graph can model the MHCRN and provide sufficient information for routing, all graph based protocols can use this graph to find the routes in a MHCRN. In the above simulations, DSDV has been used for this purpose.

\section{A. Complexity}

A graph model, while modeling the network correctly should be simple. In the following discussion the complexity of the graph model is compared with the layered graph model proposed in [6]. A sample 4 node network represented using layered graph model is reproduced from [6] in Fig. 9a. Node A has not been shown by the author for demonstration purpose. The same network is represented using multi-edged graph model in Fig. 9b. It can be said that multi-edged graph model is simpler compared to layered graph model just by visual observation. Moreover it can be observed that the layered graph model contains many unidirectional edges which add to the complexity of the graph; unlike in multi-edged model where all edges are assumed bidirectional and this is more realistic since wireless medium is bidirectional.

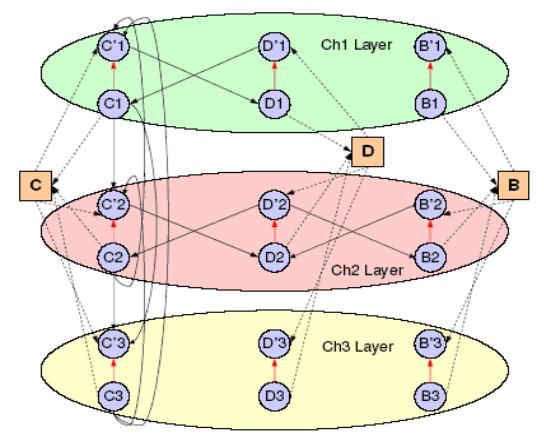

a)

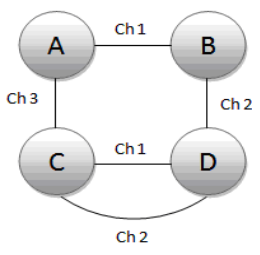

b)
Figure 9a. Layered Graph model representing a sample 4 node network. 9b. Planar Graph model representing a sample 4 node network.

Node complexity and edge complexity of the two models is given below. To understand the derivation of the complexity of the layered graph model the reader is referred to [6]. 
Let $A_{n \times n \times c}$ be a three dimensional matrix, with two dimensions representing the pair of nodes and the third dimension represents the channel in which each pair can communicate. This matrix represents all possible node pairs in all channels. The value of an element $A(x, y, z)=1$ means that nodes $x$ and $y$ can communicate using channel $z$.

Let $B_{n \times c}$ be a two dimensional matrix representing the available channels at each pair of nodes. $B(n, c)$ is 1 if $n^{\text {th }}$ node has channel $c$ available.

For a group of $n$ nodes and a total of $c$ possible channels, let $\mathrm{X}$ and $\mathrm{Y}$ be two variables defined as follows:

$$
X=\sum_{i=1}^{n} \sum_{j=1}^{n} \sum_{k=1}^{c} A(i, j, k) \text { and } Y=\sum_{j=1}^{c} B(i, j)
$$

Using these variables the layered graph model has:

$$
\begin{aligned}
& \text { Number of Nodes }=2 n c+n \\
& \text { Number of Edges }=X+\sum_{i=1}^{n}{ }^{Y} C_{2}+3 n c
\end{aligned}
$$

The planar graph model has:

$$
\begin{aligned}
& \text { Number of Nodes }=n \\
& \text { Number of Edges }=X
\end{aligned}
$$

In the planar graph model, the elements of matrix A represent the edges since $A(x, y, z)=1$, represents a possible communication between node pairs $x$ and $y$ through channel $z$. Hence, sum of all elements in matrix A, which is defined as $X$, is equal to the number of edges in multi-edged graph model. The number of nodes is equal to $n$ since there is only one layer. For example, in a set of 5 possible channels and 10 nodes, the layered graph model has 110 nodes and 258 edges whereas the multiedged graph model has only 10 nodes and 30 edges. Thus, the multi-edged graph model is much simpler than the layered graph model.

\section{CONCLUSION}

In this paper a new multi-edged graph to model a MHCRN is presented. The proposed graph model has bidirectional edges and a planar structure like in classical graph model. Using such a graph to model an MHCRN enables the direct use of traditional routing protocols for route discovery. The model is validated using a custom simulator built in C. DSDV routing protocol is used in the simulations. Finally, the node and edge complexity of the proposed model is demonstrated to be lesser than layered graph model.

\section{REFERENCES}

[1] Q. Zhao, B.M. Sadler, "A Survey of Dynamic Spectrum Access: Signal Processing, Networking, and Regulatory Policy," IEEE Signal Processing Magazine, May, 2007.

[2] C. E. Perkins, E. M. Royer, and S. R. Das, .Ad Hoc OnDemand Distance Vector (AODV) Routing, IETF Mobile Ad Hoc Networks Working Group, IETF RFC 3561.

[3] C. E. Perkins and P. Bhagwat, .Highly dynamic destinationsequenced distance-vector routing (DSDV) for mobile computers. In Proceedings of the ACM Special Interest Groupon Data Communications (SIGCOMM), August 1994, pages 234-244.

[4] D. B. Johnson, D. A. Maltz, and Y-C Hu., .The Dynamic Source Routing Protocol for Mobile Ad Hoc Networks (DSR),. IETF Mobile Ad Hoc Networks Working Group, Internet Draft, work in progress, 24 February 2003.

[5] Pradeep Kyasanur, Nitin H. Vaidya, "Protocol Design Challenges for Multi-hop Dynamic spectrum Access Networks", Proc. IEEE DySPAN 2005, November 2005, pp. 645- 648 .

[6] Chunsheng Xin, Bo Xie, Chien-Chung Shen, "A novel layered graph model for dynamic spectrum access networks”, Proc. IEEE DySPAN 2005, November 2005, pp. 308-317.

[7] Bing Qi, Saad Biaz, Shaoen Wu, Yiming Ji: An interferenceaware routing metric in multi-radio multi-hop networks. ACM Southeast Regional Conference 2007: 549-500.

[8] L. De Nardis and M.-G. Di Benedetto, "Cognitive routing in UWB networks," invited paper at the IEEE International Conference on UWB 2006 (ICUWB2006), September 24 272006. 